\title{
Índices de selección para la mejora genética de vacas Siboney de Cuba
}

\author{
Hernández, A. ${ }^{@}$ y Ponce de León, R.
}

Instituto de Ciencia Animal. Mayabeque. Cuba.

\section{PALABRAS CLAVE ADICIONALES}

Ganado de leche.

Parámetros genéticos.

Selección multicarácter.

\section{RESUMEN}

Se utilizaron los datos fenotípicos y genealógicos de vacas Siboney de Cuba (5/8 Hostein 3/8 Cebú) con partos de los años 1984 al 2016. El objetivo del estudio fue estimar los parámetros y valores genéticos (VG) de rasgos de producción, reproducción y longevidad, y construir índices de selección (IS) mediante el análisis de componentes principales (CP). Se estimaron los VG de 6425 vacas para la producción de leche acumulada hasta los 305 días $\left(\mathrm{VGL}_{305}\right)$, duración de la lactancia $\left(\mathrm{NG}_{\mathrm{D}}\right)$, edad al primer parto $\left(N G_{\text {Ep }}\right)$, intervalo parto gestación $\left(N G_{\mathbb{P G}}\right)$, leche acumulada por vida $\left(N G_{\mathbb{T V}}\right)$ y vida productiva $\left(V_{\mathrm{VP}}\right)$. Las h2 para L305, DL, EP1, IPG, LTV y VP fueron de $0.16 \pm 0.01,0.04 \pm 0.01,0.04 \pm 0.01$, $0.44 \pm 0.02,0.05 \pm 0.01$ y $0.01 \pm 0.01$, respectivamente. La correlación genética entre $L 305$ y DL fue de 0.36 , entre EP1, IPG y LTV de $0.36,-0.42$ y -0.36 , y de VP con los otros rasgos fueron bajas $1-0.24$ a 0.11 ). El $70.8 \%$ de la varianza total de los VG fue explicada por los $\mathrm{CP}_{13,3}$, siendo el CP1 el de mayor aporte (32.4\%). Los CP permitieron seleccionar por los $\mathrm{CP}_{1}$ y $\mathrm{CP}_{2}$, en lugar de seis VG. Se concluye que en el Siboney de Cuba es posible construir IS basados en los $C P$. La selección para $V_{G} L_{305}$ y VG podría realizarse mediante el índice $P C_{1}$, otro IS $\left(P C_{2}\right)$ consideraría los $V G_{\mathbb{P G}}, V_{E P 1}$ y $V G_{\mathbb{I V}}$; mientras que la VP podría mejorarse independientemente de los otros rasgos utilizando su VG.

\section{Selection index for genetic improvement of Siboney de Cuba cows}

\section{SUMMARY}

\section{ADDITIONAL KEYWORDS}

Dairy cattle.

Genetic parameter.

Multitrait selection.

\section{INFORMATION}

\section{Cronología del artículo.}

Recibido/Received: 07.06.2018

Aceptado/Accepted: 02.12.2019

On-line: 15.01.2020

Correspondencia a los autores/Contact e-mail:

arelishdez@ica.co.cu

\begin{abstract}
Phenotypic and genealogy date of Siboney de Cuba cows from 1984 to 2016 calving years was used. The goal of this study was to estimate the parameters and breeding values (NG) of production, reproduction and longevity traits. Besides to construct selection index (IS) using principal componente (CP) analysis. The VG of 6425 cows were estimated for milk yield up to 305 days $\left(V^{2305}\right)$, lactation length $\left(N G_{D}\right)$, age at first calving $\left(N G_{E P 1}\right)$, calving gestation interval $\left(N G_{\mathbb{P G}}\right)$, lifetime yields $\left(N G_{\mathbb{I V}}\right)$ y productive life $\left(N G_{V P}\right)$. The heredabilities for $L 305, D L, E P 1, I P G, L T V$ VP were of $0.16 \pm 0.01,0.04 \pm 0.01,0.04 \pm 0.01$, $0.44 \pm 0.02,0.05 \pm 0.01$ y $0.01 \pm 0.01$, respectively. The genetic correlation between $\mathrm{L} 305$ and $\mathrm{DL}$ was of 0.36 , between EP1, IPG and LTV of $0.36,-0.42$ and -0.36 , and the VP with the others traits were low $(-0.24$ to 0.11$)$. The $70.8 \%$ of the total variance of $\mathrm{VG}$ was explained for the $\mathrm{CP}_{1.3}$, doing the highest importance the $\mathrm{CP} 1(32.4 \%)$. Using $\mathrm{CP}$, it is possible to select animals based on only two scores generated by $\mathrm{PC}_{1}$ and $\mathrm{PC}_{2}$ instead of the six VG. It was concluded that it is possible to construct IS based on $\mathrm{CP}$ in Siboney de Cuba bovine. Selection for $V G_{1305}$ and $V G_{D L}$ with $P C_{1}$ index could be done separately from selection for $V G_{\mathbb{P G}}, V G_{E P 1}$ and $V G_{\mathbb{I V}}$ using $P C_{2}$ index, and the $V P$ could be improved independent of the other traits using their VG.
\end{abstract}

\section{INTRODUCCIÓN}

La selección genómica es la herramienta de mayor impacto en la eficiencia de la selección animal (Cantet, Gualdrón \& Munilla 2008). Sin embargo, aún subsisten interrogantes en cuanto a la modelación de la varianza aditiva, cuando se estima el valor de cría genómico y en la eficacia en la respuesta a la selección a largo plazo. Por otra parte, las condiciones productivas y socioeconómicas de muchos países, requieren de la utilización de los métodos convencionales de selección, como condición previa a las evaluaciones genómicas.

Con los métodos tradicionales, en el ganado lechero cubano, actualmente la selección se basa en una sola característica, la producción lechera. No obstante, en razas como el Siboney de Cuba recientemente se ha apreciado un detrimento en el comportamiento reproductivo, con promedios de 215 y 490 días de intervalo parto gestación e intervalo entre partos, respectivamente (Hernández \& Ponce de León 2016). El mismo pudiera deberse, entre otras causas, a la selección man- 
tenida por producción de leche, pues varios estudios han manifestado que la selección solo por producción de leche causa efectos negativos en la salud de la ubre (Heringstad et al. 2003) y el comportamiento reproductivo (Haile-Mariam, Bowman \& Goddard 2003; Kadarmideen et al. 2003). De ahí la necesidad de seleccionar por varios rasgos.

Los índices de selección tradicionales (Smith 1936, Hazel 1943) son utilizados para seleccionar los animales por combinaciones lineales de los valores genéticos con los pesos económicos. Sin embargo, en ocasiones resulta difícil obtener los pesos económicos. Es por ello, que países como Brasil y la India, incorporaron el análisis de componentes principales en la construcción de índices de selección del ganado lechero (Bignardi et al. 2012; Khan et al. 2013).

Según Buzanskas et al. (2013) cuando se utiliza este enfoque, los animales se pueden seleccionar de una manera equilibrada una vez que los puntajes de cada componente principal son combinaciones lineales de todos los valores genéticos de los rasgos evaluados, y no pesos empíricos generalmente utilizados en programas de mejora.

Por ello, la presente investigación se propuso como objetivo estimar los parámetros y valores genéticos (VG) de rasgos de producción, reproducción y longevidad, y construir índices de selección (IS) mediante el análisis de componentes principales, en vacas Siboney de Cuba.

\section{MATERIAL Y MÉTODOS}

Se utilizó la información de la producción lechera y la reproducción, que se registró en el Sistema de Control Pecuario (SISCOP), de las vacas Siboney de Cuba (5/8 Hostein 3/8 Cebú) que tuvieron sus partos entre los años 1984 al 2016. Estas vacas se ubicaron en tres ganaderías (Empresa Pecuaria Genética de Matanzas en la provincia de Matanzas, Los Naranjos en Mayabeque y Camilo Cienfuegos de Pinar del Río) de la región occidental de la República de Cuba.

El sistema de alimentación de las vacas fue basado en pastoreo. Las vacas lactantes consumieron fundamentalmente pasto Estrella (Cynodon nlemfuensis), pasto Pangola (Digitaria decumbens), pasto Guinea ( $\mathrm{Pa}-$ nicum maximum) y algunas especies de pasto naturales. Después del año 2000 se comenzó la introducción del Penisetum purpureum var. Cuba CT-115, con destino al pastoreo y como reserva estratégica de los períodos de sequía. A partir del año 2001 se comenzó a ofrecer DDGS (Granos secos deshidratados con solubles) a razón de $0.46 \mathrm{~kg}$ / litro desde el segundo litro producido, aproximadamente. Durante el período poco lluvioso se suministró suplementación de caña de azúcar (Saccharum officinarum), King Grass (Penisetum purpureum) en forma de forraje y hollejo de cítrico, según disponibilidad. La urea y las sales minerales formaban parte de los alimentos complementarios según lo requería la dieta.

Las hembras no lactantes se mantuvieron en las mismas condiciones de pastoreo. A los treinta días preparto recibieron suplementación con concentrado, según disponibilidad, no sobrepasando de 2-3 kg/ vaca/día. Durante el período de 1991 al 2000, no se ofreció ningún suplemento a las vacas preparto. Se realizó el ordeño mecánico 2 veces al día. Los intervalos entre ordeños fueron de 10 y 14 horas. Los horarios de pastoreo en las principales empresas estudiadas fueron los denominados normales (mañana, tarde y noche).

Se utilizaron los registros de los animales vivos y también los de aquellos que había causado baja. A partir del fichero de datos de la producción lechera (vivas + bajas) se obtuvieron los rasgos: Producción de leche acumulada hasta los 305 días (L305), duración de la lactancia (DL) y edad al primer parto (EP1). Mientras que, del fichero de datos de la reproducción (vivas + bajas) se calculó el intervalo parto gestación (IPG).

Del fichero de bajas de la producción lechera se calculó la leche acumulada por vida (LTV); así como el rasgo de longevidad denominado vida productiva (VP) determinada como los meses del primer al último parto.

\section{EDICIÓN DE LOS DATOS Y GRUPOS DE CONTEMPORÁNEOS}

Para garantizar una mayor precisión en la estimación de los parámetros genéticos, se eliminaron aquellos datos con bajas frecuencias, como las lactancias con menos de 30 días, las edades al primer parto inferiores a los 24 meses, las producciones de leche con menos de $100 \mathrm{~kg}$, y los IPG inferiores a los 24 días. Las lactancias superiores a la décima se agruparon en esta última.

Se utilizó el método de mínimos cuadrados en un modelo lineal general (GLM) mediante el paquete estadístico SAS (2010) versión 9.3 (SAS Institute, Cary, NC, USA) para definir los efectos fijos significativos $(\mathrm{P}<0.01)$ a incluir en el grupo de contemporáneos $(\mathrm{GC})$. Los GC se definieron para identificar un grupo de animales que son del mismo sexo, más o menos de la misma edad y que estuvieron en las mismas condiciones ambientales y de manejo durante una fase particular de su vida (Van Vleck 1987).

Se consideró la combinación de rebaño-año-época de parto, como GC y se eliminaron aquellos grupos conformados por menos de 3 animales. Se determinaron dos épocas de parto: la lluviosa (de mayo a octubre) y la poco lluviosa (de noviembre a abril).

El fichero de pedigrí quedo conformado por un total de 153963 individuos. La información del pedigrí alcanzó hasta los abuelos, por la línea materna y por la paterna. En la Tabla I se muestra el número de observaciones utilizado en cada fichero de datos, la cantidad de animales (toros, vacas), el número de grupos de contemporáneos (GC), los años de parto (AP), las medias y desviaciones estándar para cada rasgo.

\section{ESTIMACIÓN DE PARÁMETROS GENÉTICOS}

Se empleó el programa ASREML (Gilmour et al. 2003) para estimar los componentes de varianza, heredabilidades $\left(\mathrm{h}^{2}\right)$ y valores genéticos (VG) mediante un modelo animal unicarácter, cuyos efectos variaron de acuerdo a los rasgos estudiados.

Se empleó el siguiente modelo: $\mathrm{y}=\mathrm{Xb}+\mathrm{Za}+\mathrm{e}$ 
Tabla I. Número de observaciones (N), años de parto (AP), media y desviación estándar (DE) en rasgos de la producción de leche, la reproducción y la longevidad de vacas Siboney de Cuba (Number of observations (N), calving years (AP), mean and standard deviation (DE) of milk yield, reproduction and longevity traits of Siboney de Cuba cows).

\begin{tabular}{lccccccc}
\hline Rasgos & $\mathrm{N}$ & Vacas & Toros & GC & AP & Media & DE \\
\hline L305 (kg) & 47567 & 17235 & 348 & 3292 & $1987-2016$ & 1545.55 & 805.62 \\
DL (días) & 47567 & 17235 & 348 & 3292 & $1987-2016$ & 271.89 & 83.07 \\
LTV (kg) & 13171 & 13171 & 339 & 2553 & $1984-2015^{*}$ & 3894.70 & 3741.95 \\
EP1(meses) & 14383 & 14383 & 332 & 1899 & $1987-2016^{*}$ & 45.10 & 15.07 \\
IPG (días) & 47032 & 14118 & 313 & 3915 & $1986-2016$ & 232.51 & 170.70 \\
VP (meses) & 8437 & 8437 & 228 & 1586 & $1984-2014^{*}$ & 47.46 & 26.77 \\
\hline
\end{tabular}

L305: Producción de leche acumulada hasta los 305 días; DL: Duración de la lactancia; LTV: Leche acumulada por vida; EP1: Edad al primer parto; IPG: Intervalo parto gestación; VP: Vida productiva *Años de primer parto.

Donde y: es el vector de las observaciones para los rasgos EP1, LTV y VP; b: vector de efectos fijos (contienen el efecto de rebaño-año-época de primer parto y la edad al primer parto como covariable lineal y cuadrática), a: vector de efectos aleatorios del animal, $X$ y $Z$ son las matrices de diseño o de incidencia (relacionan a los efectos fijos y aleatorios con los datos, respectivamente) y e: vector de efectos residuales aleatorios.

El otro modelo utilizado fue uno de repetibilidad:

$$
\mathrm{y}=\mathrm{Xb}+\mathrm{Za}+\mathrm{Wp}+\mathrm{e}
$$

Donde y:es el vector de las observaciones para los rasgos L305, DL, e IPG; b: vector de efectos fijos que contienen el efecto de rebaño-año-época de parto y la edad al parto como covariable lineal y cuadrática, a: vector de efectos aleatorios del animal, p: vector del efecto del ambiente permanente de la vaca, X, Z y $\mathrm{W}$ son las matrices de diseño o de incidencia que relacionan a los efectos fijos, aleatorios y del ambiente permanente con los datos, respectivamente y e: vector de efectos residuales aleatorios.

Para estimar las correlaciones genéticas y ambientales, se empleó el siguiente modelo animal multicarácter:

$$
\left[\begin{array}{l}
y_{1} \\
y_{2} \\
y_{3} \\
y_{4} \\
y_{5} \\
y_{5}
\end{array}\right]=\left[\begin{array}{cccccc}
x_{1} & 0 & 0 & 0 & 0 & 0 \\
0 & x_{2} & 0 & 0 & 0 & 0 \\
0 & 0 & x_{3} & 0 & 0 & 0 \\
0 & 0 & 0 & x_{4} & 0 & 0 \\
0 & 0 & 0 & 0 & x_{5} & 0 \\
0 & 0 & 0 & 0 & 0 & x_{6}
\end{array}\right]\left[\begin{array}{l}
b_{1} \\
b_{2} \\
b_{3} \\
b_{4} \\
b_{5} \\
b_{6}
\end{array}\right]+\left[\begin{array}{cccccc}
z_{1} & 0 & 0 & 0 & 0 & 0 \\
0 & z_{2} & 0 & 0 & 0 & 0 \\
0 & 0 & z_{3} & 0 & 0 & 0 \\
0 & 0 & 0 & z_{4} & 0 & 0 \\
0 & 0 & 0 & 0 & z_{5} & 0 \\
a_{2} \\
a_{3} \\
a_{4} \\
a_{5} \\
a_{3} & 0 & 0 & 0 & 0 & z_{0}
\end{array}\right]+\left[\begin{array}{l}
\mathrm{e}_{1} \\
\mathrm{e}_{2} \\
\mathrm{e}_{3} \\
\mathrm{e}_{4} \\
\mathrm{e}_{5} \\
\mathrm{e}_{6}
\end{array}\right]
$$

Donde:

$\mathrm{y}_{\mathrm{i}}=$ vector de las observaciones para el $i$-ésimo rasgo

$b_{i}=$ vector de los efectos fijos (rebaño-año-época de parto y la edad al parto como covariable lineal y cuadrática) para el i-ésimo rasgo

$\mathrm{a}_{\mathrm{i}}=$ vector de los efectos aleatorios del animal para el $i$-ésimo rasgo

$\mathrm{e}_{\mathrm{i}}=$ vector de los efectos residuales aleatorios para el $i$-ésimo rasgo

$\mathrm{X}_{\mathrm{i}} \mathrm{y} \mathrm{Z}_{\mathrm{i}}=$ matrices de diseño que relacionan los datos con los efectos fijos y aleatorios, respectivamente

Se asume que:

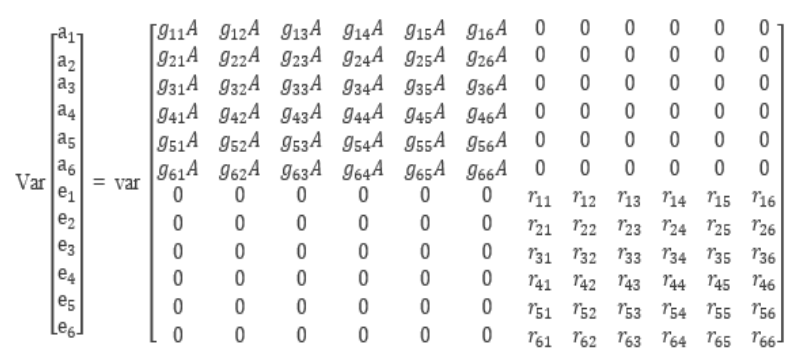

Donde: $\mathrm{g}_{\mathrm{ij}}=$ varianza genética aditiva para el $i$-ésimo rasgo, cuando $\mathrm{i}=\mathrm{j}$, la covarianza genética cuando $\mathrm{i} \neq \mathrm{j}$; $\mathrm{A}=$ matriz de relaciones y $\mathrm{r}_{\mathrm{ij}}=$ varianza residual cuando $\mathrm{i}=\mathrm{j}$, la covarianza cuando $\mathrm{i} \neq \mathrm{j}$.

\section{ANÁLISIS DE COMPONENTES PRINCIPALES}

Se utilizaron los VG estimados para cada uno de los rasgos, mediante el modelo animal unicarácter. Se importaron al paquete estadístico SAS (2010) versión 9.3 (SAS Institute, Cary, NC, USA) y posteriormente se unieron todos los ficheros dejando solo aquellas vacas que tuvieron información de los VG para todos los rasgos estudiados. El fichero final presentó un total de 6425 vacas.

El análisis de componentes principales (CP) se realizó mediante el paquete estadístico SPSS (2002) versión 11.5. Este análisis se realizó como una vía para condensar o resumir la información contenida en varias variables originales (en este caso los VG) en un conjunto más pequeño de nuevas dimensiones compuestas o variantes llamadas componentes principales, con una pérdida mínima de información, y para explorar las relaciones entre los VG obtenidos del análisis unicarácter $\mathrm{VG}_{\mathrm{L} 305}, \mathrm{VG}_{\mathrm{DL}}, \mathrm{VG}_{\mathrm{LTV}}, \mathrm{VG}_{\mathrm{EP} 1}, \mathrm{VG}_{\mathrm{IPG}}, \mathrm{VG}_{\mathrm{VP}}$, ) para explicar la estructura de los datos (Hair et al. 2009).

Debido a las diferencias en las unidades de medida, los VG para todos los rasgos se estandarizaron usando la distribución normal estándar (' $z$ '). Se utilizó el criterio de Kaiser (1960) para seleccionar el componente principal que explica la mayor variación genética de los datos. Este criterio toma en consideración solo aquellos componentes principales con valores propios por encima de la unidad. El valor propio de un componente principal está asociado con la varianza de todos los rasgos incluidos en el componente principal. 
Cada valor propio está asociado con un vector unitario llamado vector propio (Rencher 2002). Los vectores propios representan la fortaleza y dirección de la varianza de cada rasgo con el componente principal. En el presente trabajo se utilizó una matriz de correlación de variables para obtener valores propios. El primer componente principal explica el más amplio porcentaje de la varianza total de los VG. El segundo explica el segundo porcentaje más amplio, etc; hasta que toda la varianza de la base de datos sea explicada.

Al utilizar los VG estandarizados en este análisis, cada componente principal puede generar un nuevo valor denominado puntuación del componente principal, el cual es la suma de los VG estandarizados del peso de cada rasgo por su respectivo coeficiente de puntuación estandarizado (SSC). De esta forma, el componente principal puede ser usado como un índice para evaluar animales, para múltiples rasgos. Los coeficientes de puntuación estandarizados de cada VG en cada componente principal se obtuvieron utilizando la siguiente formula:

$$
\mathrm{SSC}_{\mathrm{ij}}=\frac{\text { vector propio } \mathrm{ij}}{\sqrt{\text { valor propio }}_{j}}
$$

Donde:

$\mathrm{SSC}_{\mathrm{ij}}=$ Coeficientes de puntuación estandarizados de los VG de cada rasgo en el j-ésimo componente principal

La puntuación del componente principal (índice) se calculó como:

$$
\mathrm{CP}_{\mathrm{jl}}=\sum \text { SSCij VGil }
$$

\section{Donde:}

$\mathrm{CP}_{\mathrm{jl}}=$ Puntuación del componente principal (puntuación del índice) para el l-ésimo animal en el j-ésimo componente principal

$S C_{i j}=$ Coeficientes de puntuación estandarizados de los VG del i-ésimo rasgo en el j-ésimo componente principal
$\mathrm{VG}_{\mathrm{il}}=$ Valor genético estimado estandarizado del i-ésimo rasgo en el l-ésimo animal

\section{RESULTADOS Y DISCUSIÓN}

\section{ESTIMACIÓN DE PARÁMETROS GENÉTICOS}

Los estimados de las heredabilidades y correlaciones genéticas y ambientales se presentan en la Tabla II. Se espera obtener mayores mejoras en el rebaño para los rasgos producción de leche acumulada hasta los 305 días y edad al primer parto, si se incluyeran en un programa de selección, dado que fueron los que presentaron mayores estimados de heredabilidad.

La heredabilidad de la producción de leche acumulada hasta los 305 días se corresponde con los estimados en zonas tropicales, donde presentan un rango más bajo. Desde valores de 0.19 y 0.22 en razas como la Carora (5/8 Pardo Suizo 3/8 Criollo) en Venezuela (Valle \& Moura 1986; Cerutti et al. 1994), 0.19 en vacas de la raza Gir en Brasil (Ledic et al. 2002) y en el Mambí de Cuba (Hernández et al. 2011a), hasta estimados de 0.17 y 0.18 en el propio Siboney de Cuba, en otras ganaderías (Ribas et al. 2004). En rebaños de vacas Holstein cubanas también se obtuvo un estimado de 0.16 usando un modelo de repetibilidad (Palacios-Espinosa et al. 2007).

Autores como Amimo et al. (2007) plantearon que este comportamiento no era únicamente causado por el efecto directo del clima, sino que eran de mayor importancia los efectos de la poca calidad de los forrajes, el bajo consumo de concentrado, la alta incidencia de parásitos y enfermedades y la pobre economía e infraestructura.

Altas heredabilidades para la edad al primer parto también fueron referidas por Ruiz-Sánchez et al. (2007), Vercesi Filho et al. (2007) y Ríos-Utrera et al. (2013) en ganado Holstein e Indubrasil quienes encontraron estimados de $0.47,0.48$ y 0.39 , respectivamente. Estos valores indican que esta característica puede disminuirse mediante selección. En estudios previos en vacas Siboney de Cuba se encontraron variaciones en los estimados de heredabilidad con valores de 0.15 (Suárez \& Pérez 2005), 0.31 (Portales et al. 2007) y 0.05 (Portales et al. 2009). Las diferencias encontradas en relación con los estimados de la heredabilidad pueden

Tabla II. Estimados de heredabilidad (en la diagonal), correlaciones genéticas (arriba de la diagonal) y ambientales (debajo de la diagonal) en rasgos de la producción de leche, la reproducción y la longevidad de vacas Siboney de Cuba (Estimators of heredability (in the diagonal), genetic (above the diagonal) and environment correlation (below the diagonal) of milk yield, reproduction and longevity traits of Siboney de Cuba cows).

\begin{tabular}{lcccccc}
\hline Rasgos & L305 & DL & LTV & EP1 & IPG & VP \\
\hline L305 & $0.16 \pm 0.01$ & $0.36 \pm 0.01$ & $0.14 \pm 0.01$ & $-0.01 \pm 0.01$ & $-0.11 \pm 0.01$ & $-0.09 \pm 0.01$ \\
DL & $0.65 \pm 0.01$ & $0.04 \pm 0.01$ & $0.34 \pm 0.02$ & $0.24 \pm 0.01$ & $0.14 \pm 0.01$ & $-0.13 \pm 0.02$ \\
LTV & $0.36 \pm 0.01$ & $0.24 \pm 0.01$ & $0.04 \pm 0.01$ & $-0.42 \pm 0.01$ & $-0.36 \pm 0.01$ & $0.11 \pm 0.02$ \\
EP1 & $0.01 \pm 0.01$ & $0.02 \pm 0.01$ & $-0.09 \pm 0.01$ & $0.44 \pm 0.02$ & $0.36 \pm 0.02$ & $-0.24 \pm 0.02$ \\
IPG & $0.07 \pm 0.01$ & $0.17 \pm 0.01$ & $-0.10 \pm 0.01$ & $-0.03 \pm 0.01$ & $0.05 \pm 0.01$ & $-0.15 \pm 0.01$ \\
VP & $0.10 \pm 0.01$ & $0.10 \pm 0.01$ & $0.75 \pm 0.01$ & $-0.13 \pm 0.01$ & $0.05 \pm 0.01$ & $0.01 \pm 0.01$ \\
\hline
\end{tabular}

L305: Producción de leche acumulada hasta los 305 días; DL: Duración de la lactancia; LTV: Leche acumulada por vida; EP1: Edad al primer parto; IPG: Intervalo parto gestación; VP: Vida productiva 
Tabla III. Valores propios para cada componente principal, proporción total y acumulada de la varianza genético aditiva, del primer al sexto componente principal $\left(\mathrm{CP}_{1_{6}}{ }_{6}\right)$, en vacas Siboney de Cuba (Eigenvalues for each principal component, total and cumulative proportion of additive genetic variance, of first to sixth principal components $\left(\mathrm{CP}_{1-6}\right)$, of Siboney de Cuba cows).

\begin{tabular}{lccc}
\hline Componentes & Total & \% de la varianza genético aditiva & \% acumulado \\
\hline $\mathrm{CP}_{1}$ & 1.945 & 32.414 & 32.414 \\
$\mathrm{CP}_{2}$ & 1.252 & 20.862 & 53.276 \\
$\mathrm{CP}_{3}$ & 1.055 & 17.585 & 70.861 \\
$\mathrm{CP}_{4}$ & 0.765 & 12.743 & 83.604 \\
$\mathrm{CP}_{5}$ & 0.662 & 11.038 & 94.642 \\
$\mathrm{CP}_{6}$ & 0.321 & 5.358 & 100.000 \\
\hline
\end{tabular}

estar relacionadas con la desviación de la media del rebaño, la cantidad total de datos a evaluar, la forma de la edición de los datos, entre otros.

Los estimados de heredabilidad para el IPG fueron bajos y coinciden con los obtenidos en otras razas como el Cebú y la Santa Gertrudis, en Cuba (Santana et al. 2004; Veliz et al. 2004; Guerra-Iglesias et al. 2009). Los resultados anteriores indican que este rasgo se encuentra influenciado por el ambiente, y por consiguiente su mejoramiento, en dichos rebaños, debe realizarse a través de la optimización de las condiciones de alimentación, sanidad y manejo reproductivo del sistema de producción.

Campos et al. (1994) en Colombia informaron que las características relacionadas con el desempeño reproductivo como el período abierto (IPG) y el intervalo entre partos (IPP) se caracterizaban porque los valores encontrados para sus parámetros genéticos eran generalmente bajos, con valores de heredabilidad inferiores al $10 \%$, en la mayoría de los casos.

Según Grossi et al. (2008) la baja variabilidad encontrada en los rasgos reproductivos puede atribuirse a que algunos productores especifican empíricamente una edad o un peso para comenzar la reproducción de las novillas, por lo tanto, mucha de la variación se pierde y las hembras precoces sexualmente no se pueden detectar.

Las correlaciones genéticas entre la leche acumulada hasta 305 días y la duración de la lactancia fueron moderadas por lo que la selección por producción de leche también mejorará, de alguna forma, la duración de la lactancia. Mientras que, la correlación genética de la leche acumulada hasta 305 días con el resto de los rasgos estudiados (LTV, EP1, IPG y VP) fue baja lo cual implica que estos rasgos cambiarán casi independientemente de la producción lechera.

Los resultados anteriores indican que las vacas con mayores producciones acumuladas hasta 305 días no fueron las que más produjeron durante toda su vida, y tampoco fueron las más longevas. En vacas Mambí de Cuba (Hernández et al. 2011b) se apreciaron resultados similares con estimados negativos y bajos entre la leche producida en primera lactancia y la vida productiva (-0.16) y aquel entre la leche producida en primera lactancia y la leche acumulada por vida fue de 0.07. Este comportamiento probablemente se deba a que no hubo un tratamiento diferencial para las vacas altas productoras, pues la cantidad de concentrado que se les ofrece está en correspondencia con la media del grupo de alta, que es inferior a su promedio de producción lechera, y por consiguiente no cubren sus requerimientos nutricionales. Esto puede provocar problemas reproductivos y ser las más propensas a causar baja.

La edad al primer parto presento una correlación moderada y positiva con el intervalo parto gestación. Mientras que, las correlaciones genéticas de la leche acumulada por vida con la edad al primer parto y el intervalo parto gestación fueron moderadas y antagónicas por lo que al disminuir la edad al iniciar el parto también disminuirán los intervalos reproductivos y se espera que en cierta medida aumente la producción lechera acumulada por vida.

Varios autores hallaron un efecto de la edad al primer parto en los rasgos de producción lechera, longevidad y de reproducción (Ojango \& Pollot 2001; Hare, Norman \& Wright 2006; Ben Gara et al. 2009; Castillo et al. 2013) del ganado lechero. En Estados Unidos los hatos donde las novillas parieron a una edad avanzada, produjeron menos leche (Bewley, Palmer \& Jackson 2001). Se reportó que el efecto de la edad al primer parto en la longevidad y el rendimiento de la vida productiva del animal, era maximizado con una edad de 20 a 36 meses en la primera lactancia (Ettema \& Santos 2004; Ojango, Ducrocq \& Pollott 2005; Ruiz-Sánchez et al. 2007). Mientras que, Galvis (2008) indicó que la edad óptima para lograr un buen desempeño productivo y reproductivo era de 24 meses. Por su parte, Buzanskas et al. (2010) informaron que los animales que paren a una edad temprana tienen más probabilidades de tener un mejor rendimiento reproductivo durante un tiempo prolongado.

\section{ANÁLISIS DE COMPONENTES PRINCIPALES}

Los tres primeros componentes principales $\left(\mathrm{CP}_{1}\right.$, $\mathrm{CP}_{2} \mathrm{CP}_{3}$ ) fueron los que obtuvieron el criterio de Kaiser (Tabla III). De las 6 dimensiones originales (por ejemplo: $\left.\mathrm{VG}_{\mathrm{L} 305}, \mathrm{VG}_{\mathrm{DL}}, \mathrm{VG}_{\mathrm{LTV}}, \mathrm{VGEP}_{1}, \mathrm{VG}_{\mathrm{IPG}}, \mathrm{VG}_{\mathrm{VP}}\right)$, el $70.8 \%$ de la varianza total de los valores genéticos fue explicada por los $\mathrm{CP}_{1-3}$, siendo el $\mathrm{CP}_{1}$ el de mayor aporte pues explicó el $32.4 \%$ de la varianza total.

Estos resultados se corresponden con otras investigaciones que evidenciaron la utilidad del análisis de CP para reducir la dimensionalidad de los rasgos. En 
Tabla IV. Correlaciones lineales de los VG estandarizados con el componente principal 1 al 3 $\left(\mathrm{CP}_{1-3}\right)$, en vacas Siboney de Cuba (Linear correlations of standardised breeding values with one to the three principal component $\left(\mathrm{CP}_{1-3}\right)$ of Siboney de Cuba cows).

\begin{tabular}{lccc}
\hline VG & \multicolumn{3}{c}{ Componentes } \\
& $\mathrm{CP}_{1}$ & $\mathrm{CP}_{2}$ & $\mathrm{CP}_{3}$ \\
\hline $\mathrm{VG}_{\mathrm{L305}}$ & 0.867 & -0.100 & 0.004 \\
$\mathrm{VG}_{\mathrm{DL}}$ & 0.881 & -0.005 & -0.043 \\
$\mathrm{VG}_{\mathrm{LTV}}$ & 0.405 & 0.586 & -0.320 \\
$\mathrm{VG}_{\mathrm{IPG}}$ & 0.448 & -0.654 & 0.039 \\
$\mathrm{VG}_{\mathrm{VP}}$ & 0.083 & -0.015 & 0.917 \\
$\mathrm{VG}_{\mathrm{EP} 1}$ & 0.214 & 0.686 & 0.331 \\
\hline
\end{tabular}

búfalos, Oliveira et al. (2014) evaluaron siete rasgos productivos y dos reproductivos y concluyeron que el modelo de rangos reducidos con 3 o $4 \mathrm{CP}$ era suficiente para explicar el mayor porcentaje de la varianza genética aditiva de todos los rasgos. Por su parte, AgudeloGómez, Pineda-Sierra S y Cerón-Muñoz (2015) observaron que los tres primeros CP explicaron el $65.78 \%$ de la varianza original de los valores genéticos para la producción lechera a 270 días, la edad al primer parto, el peso al destete y el peso a diferentes edades (12, 18 y 24 meses) de búfalos colombianos. En vacas Holstein, Bignardi et al. (2012) apreciaron que solo se requerían dos $\mathrm{CP}$ para resumir la variación genética de los toros entre los 10 rasgos evaluados (10 producciones mensuales de leche). También en vacas Canchim, Buzanskas et al. (2013) reportaron que el 73.37\% de la varianza total de los valores genéticos de tres rasgos reproductivos y uno de crecimiento era explicada por dos CP.

Las correlaciones lineales entre los VG de los rasgos estudiados con cada componente principal (Tabla IV) mostraron que los rasgos relacionados con la producción de leche y la duración de la lactancia están altamente relacionados con la $\mathrm{CP}_{1}$ mientras que el IPG, LTV y EP1 estuvieron más asociados con la $\mathrm{CP}_{2}$. La VP estuvo relacionada con la $\mathrm{CP}_{3}$. Así el $\mathrm{CP}_{1}$ pudiera ser interpretado como un índice genético relacionado a los rasgos de producción lechera y duración de la lactancia, mientras que el $\mathrm{CP}_{2}$ pudiera ser otro índice

\begin{tabular}{|c|c|c|c|}
\hline \multicolumn{4}{|c|}{$\begin{array}{l}\text { Tabla V. Coeficientes de puntuación estandariza- } \\
\text { dos (SSC) de cadaVG estimado estandarizado para } \\
\text { todos los rasgos en cada componente principal } \\
\left(\mathrm{CP}_{1-3}\right) \text {, en vacas Siboney de Cuba (Standardised score } \\
\text { coefficients (SSC) of each of the standardised estimated breeding } \\
\text { values for all traits in each principal component }\left(\mathrm{CP}_{1-3}\right) \text {, of Siboney } \\
\text { de Cuba cows). }\end{array}$} \\
\hline \multirow[t]{2}{*}{ VG } & \multicolumn{3}{|c|}{ Coeficientes de puntuación estandarizados } \\
\hline & $\operatorname{SSC}\left(\mathrm{CP}_{1}\right)$ & $\operatorname{SSC}\left(\mathrm{CP}_{2}\right)$ & $\operatorname{SSC}\left(\mathrm{CP}_{3}\right)$ \\
\hline$V_{G_{L 305}}$ & 0.446 & -0.080 & 0.004 \\
\hline$V G_{D L}$ & 0.453 & -0.004 & -0.041 \\
\hline$V G_{\text {LTV }}$ & 0.208 & 0.469 & -0.303 \\
\hline$V_{G_{I P G}}$ & 0.230 & -0.522 & 0.037 \\
\hline$V_{\mathrm{VP}}$ & 0.043 & -0.012 & 0.869 \\
\hline$V G_{E P 1}$ & 0.110 & 0.548 & 0.313 \\
\hline
\end{tabular}

genético relacionado a la reproducción y la producción lechera por vida. En el caso del $\mathrm{CP}_{3}$ sería mejor seleccionar los animales considerando solo su VG para VP, en vez de usar un índice, dado que las correlaciones de los VG del resto de los rasgos con esta componente fueron bajas.

Los pesos de estos índices son coeficientes de puntuación estandarizados (SSC) para cada VG estandarizado (Tabla V). El mayor de los valores absolutos del SSC es el de mayor importancia relativa de los VG estandarizados en el componente principal. Esta importancia es explicada por la mayor correlación lineal entre los rasgos con el componente principal (Tabla IV).

A partir de los coeficientes de puntuación estandarizados (SSC) se calculó la puntuación del componente principal (valor del índice) para cada animal, en cada componente principal, como:

$\mathrm{CP}_{1}=0.446\left(\mathrm{VG}_{\mathrm{L} 305}\right)+0.453\left(\mathrm{VG}_{\mathrm{DL}}\right)+0.208\left(\mathrm{VG}_{\mathrm{LTV}}\right)+$ $0.230\left(\mathrm{VG}_{\mathrm{IPG}}\right)+0.043\left(\mathrm{VG}_{\mathrm{VP}}\right)+0.110\left(\mathrm{VG}_{\mathrm{EP} 1}\right)$

$\mathrm{CP}_{2}=-0.080\left(\mathrm{VG}_{\mathrm{L} 305}\right)-0.004\left(\mathrm{VG}_{\mathrm{DL}}\right)+0.469\left(\mathrm{VG}_{\mathrm{LTV}}\right)-$ $0.522\left(\mathrm{VG}_{\mathrm{IPG}}\right)-0.012\left(\mathrm{VG}_{\mathrm{VP}}\right)+0.548\left(\mathrm{VG}_{\mathrm{EP} 1}\right)$

La selección para $V_{\mathrm{L}_{305}}$ y $\mathrm{VG}_{\mathrm{DL}}$ mediante la $\mathrm{CP}_{1}$ podría realizarse por separado de la selección para $\mathrm{VG}_{\mathrm{IPG}}, \mathrm{VG}_{\mathrm{EP} 1}$ y $\mathrm{VG}_{\mathrm{LTV}}$ a través de la $\mathrm{CP}_{2}$ considerando la correlación lineal entre los VG con cada componente principal (Tabla IV). La selección para VP puede realizarse directamente por su valor genético. Estos resultados también se corresponden con las correlaciones genéticas entre los rasgos estudiados (Tabla II), que revelaron una correlación genética media entre L305 y DL (0.36) y entre EP1, IPG y LTV $(0.36,-0.42$ y -0.36$)$; mientras que las correlaciones genéticas de estos cinco rasgos con la VP fueron bajas (entre - 0.24 y 0.11 ) por lo que cambiaran casi independientemente.

Buzanskas et al. (2013) expusieron que el uso de los $\mathrm{CP}$ es una metodología para construir combinaciones lineales entre los valores genéticos de los rasgos disponibles en una base de datos, tomando en consideración los valores propios del componente principal y los vectores propios de los rasgos en cada componente principal, los cuales son medidas de variabilidad. De esta forma, rasgos con bajos estimados de heredabilidad, que raramente son tomados en consideración en un proceso de selección directa, pueden incluirse en el componente principal.

En el presente estudio, con el uso del análisis de componentes principales se pueden seleccionar los animales en base a solo dos puntajes generados por las $\mathrm{CP}_{1}$ y $\mathrm{CP}_{2}$, en lugar de los seis valores genéticos. A pesar de los bajos estimados de $h^{2}$ para L305 y DL, la CP ${ }_{1}$ puede considerarse un índice genético de producción lechera porque favorece a los animales genéticamente superiores para $\mathrm{VG}_{\mathrm{L} 305}$ y $\mathrm{VG}_{\mathrm{DL}}$; mientras que el $\mathrm{CP}_{2}$ puede ser un índice genético relacionado a la reproducción y producción lechera por vida. Los VG más relacionados con la $\mathrm{CP}_{1}$ tienen mayores varianzas que aquellos asociados con la $\mathrm{CP}_{2}$. La mayor variabilidad para $V_{\mathrm{L} 305}$ y $V_{\mathrm{DL}}$ puede explicarse por el hecho de que actualmente solo se hace énfasis en la selección por $\mathrm{VG}_{\mathrm{L} 305}$ y no se aplica una alta intensidad de selección. 
En el Siboney de Cuba es posible construir IS basados en los CP donde los $\mathrm{VG}_{\mathrm{L} 305}$ y $\mathrm{VG}_{\mathrm{DL}}$ mostraron considerable variación genética, por lo que podrían ser incluidos en un programa de selección. La selección para $\mathrm{VG}_{\mathrm{L} 305} \mathrm{y} \mathrm{VG}_{\mathrm{DL}}$ podría realizarse mediante el índice $\mathrm{PC}_{1}$, otro IS $\left(\mathrm{PC}_{2}\right)$ consideraría los $\mathrm{VG}_{\mathrm{IPG}}, \mathrm{VG}_{\mathrm{EP} 1}$ y $\mathrm{VG}_{\mathrm{LTV}}$; mientras que la VP podría mejorarse independientemente de los otros rasgos utilizando su VG.

\section{AGRADECIMIENTOS}

Este artículo hace parte del proyecto "Estrategias de selección para la mejora genética de las razas Siboney de Cuba y Mambí de Cuba" perteneciente al Programa nacional de alimento humano del Ministerio de Agricultura (MINAG) de Cuba. Se agradece a los trabajadores de las unidades de producción, en especial a los controles técnicos y especialistas en genética por su cooperación con las bases de datos e información necesaria para el desarrollo del presente trabajo.

\section{BIBLIOGRAFÍA}

Agudelo-Gómez, D, Pineda-Sierra, S\& Cerón-Muñoz,MF 2015, 'Genetic evaluation of dual-purpose buffaloes (Bubalus bubalis) in Colombia using principal component analysis', PLOS ONE, vol. 10, no. 7, pp. 1-9, viewed 6 feb 2018, <http://journals.plos.org/plosone/ article?id=10.1371/journal.pone.0132811>.

Amimo, JO, Wakhungu, JW, Inyangala, BO \& Mosi, RO 2007, 'The effects of non-genetic factors and estimation of genetic and phenotypic parameters and trends for milk yield in Ayrshire cattle in Kenya', Livestock Research for Rural Development, vol. 19, no. 1, viewed 6 feb 2018, <http://www.cipav.org.co//rrd//rrd19/1/amim 19013.htm>.

Portales, A, González-Peña, D, Guerra, D, Évora, JC \& Acosta, M 2009, 'Estimación de componentes de varianza para rasgos productivos y reproductivos en ganado Siboney de Cuba', Ciencia y Tecnología Ganadera, vol. 3, no. 1, pp. 39-44.

Ben Gara, A, Bouraoui, R, Rekik, B, Hammani, H \& Rouissi, H 2009, 'Optimal age at first calving for improved milk yield and length of productive life in Tunisian Holstein cows', American-Eurasian Journal of Agronomy, vol. 2, no. 3, pp. 162-167.

Bewley, JA, Palmer, RW \& Jackson, DB 2001, 'An overview of experiences of Wisconsin dairy farmers who modernized their operations', Journal of Dairy Science, vol. 84, no. 3, pp. 717-729.

Bignardi, AB, El Faro, L, Rosa, GJM, Cardoso, VL, Machado, PF \& Albuquerque LG 2012, Short communication. 'Principal components and factor analytic models for test-day milk yield in Brazilian Holstein cattle', Journal of Dairy Science, vol. 95, no. 4, pp. 2157-2164, viewed 21 nov 2017, <https://www.ncbi.nlm.nih.gov/pubmed/22459860>.

Buzanskas, ME, Grossi, DA, Baldi, F, Barrozo, D, Silva, LOC, Torres Junior, RAA, Munari, DP \& Alencar, MM 2010, 'Genetic associations between stayability and reproductive and growth traits in Canchim beef cattle', Livestock Science, vol. 132, pp. 107-112.

Buzanskas, ME, Savegnago, RP, Grossi, DA, Venturini, GC, Queiroz, AS, Silva, LO, Junior, RA, Munari, DP \& Alencar, MM 2013, 'Genetic parameter estimates and principal componente analysis of breeding values of reproduction and growth traits in female Canchim cattle', Reproduction, Fertility and Development, vol. 25, no. 5, pp. 775-81, viewed 21 nov 2017, <http://dx.doi.org/10.1071/RD12132>.

Campos, MS, Wilcox, CJ, Becerril, CM \& Diz, A 1994, 'Genetic parameters for yield and reproductive traits of Holstein and Jersey cattle in Florida', J Dairy Sci., vol. 77, pp. 867-873.

Cantet, RJC, Gualdrón, JL \& Munilla, S 2008,' ', Revista Argentina de Producción Animal, vol. 28, no. 2, pp. 133-136.
Castillo, G, Salazar, M, Murillo, J \& Romero JJ 2013, 'Efecto de la edad al primer parto sobre parámetros productivos en vacas Jersey de Costa Rica', Agronomía Mesoamericana, vol. 24, no. 1, pp. 177-187.

Cerutti, FM, Caroli, A, Crimella, C, Bagnato, A \& López, E 1994, 'Heredabilidad y repetibilidad de la producción de leche en la raza Carora', XIV Congreso Panamericano de Ciencias Veterinarias, Acapulco, México.

Ettema, JF \& Santos, JP 2004, 'Impact of age at calving on lactation, reproduction, health, and income in first-parity Holsteins on commercial farms', Journal of Dairy Science, vol. 87, no. 8, pp. 2730-2742.

Gilmour, AR, Gogel, BJ, Cullis, BR, Welham, SJ \& Thompson R 2003, 'ASREML'. UserGuide Release 1.10. <https://www.vsni.co.uk/ downloads/asreml/release 1/doc/UserGuide.pdf>.

Goyache, F, Gutiérrez, JP, Fernández, I, Royo, U \& Álvarez, I 2005, 'Genetic analysis of days open in beef cattle', Short communication, Livestock Production Science, vol. 93, pp. 283-289, viewed 21 nov 2017,< https:// www.elsevier.com/locate/livprodsci>.

Grossi, DA, Frizzas, OG, Paz, CCP, Becerra, LAF, Lobo, RB, Oliveira, JA \& Munari, DP 2008, 'Genetic associations between acumulated productivity and reproductive and growth traits in Nerole cattle', Livestock Science, vol. 117, pp. 139-146.

Guerra-Iglesias, D, Espinoza-Villavicencio, JL, Palacios-Espinosa, A, González-Peña, D, Rodríguez-Almeida, F, Guillén-Trujillo, A 2009, 'Componentes de (co)varianza de los días abiertos en bovinos Santa Gertrudis', Técnica Pecuaria en México, vol. 47, viewed 18 Jan 2018, <http://www.redalyc.org/articulo.oa?id=61312116003>.

Haile-Mariam, M, Bowman, PJ \& Goddard, ME 2003, 'Genetic and environmental relationships among calving interval, survival, persistency of milk yield and somatic cell count in dairy cattle', Livestock Production Science, vol. 80, pp. 189-200.

Hair, JF, Black, WC, Babin, BJ \& Anderson, RE 2009, 'Multivariate data analysis', (Prentice Hall: Upper Saddle River, NJ.).

Hare, E, Norman, HD \& Wright, JR 2006, 'Trends in Calving Ages and Calving Intervals for Dairy Cattle Breeds in the United States', Journal of Dairy Science, vol. 89, no. 1, pp. 365-370.

Hazel, LN 1943, 'The genetic basis for constructing selection indexes', Genetics, vol. 28, pp. 476-490.

Heringstad, B, Chang, YM, Gianola, D \& Klemetsdal, G 2003, 'Genetic analysis of longitudinal trajectory of clinical mastitis in first-lactation Norwegian Cattle', Journal of Dairy Science, vol. 86, pp. 2676-2683.

Hernández, A\& Ponce de León, R 2016, 'Milk production and reproduction in cows with different proportions of Bos Taurus $x$ Bos indicus genes', Cuban Journal of Agricultural Science, vol. 50, no. 2, pp. 193-203.

Hernández, A, Ponce de León, R, García, SM, Guzmán, G \& Mora, M 201 la, 'Genetic assessment of the dairy cattle Mambí de Cuba', Cuban Journal of Agricultural Science, vol. 45, no. 4, pp. 355-359.

Hernández, A, Ponce de León, R, Gutiérrez, M, García, R, García, SM, Mora, M \& Guzmán, G 201 1b, 'Parámetros genéticos en rasgos de la producción lechera y la longevidad de vacas Mambí de Cuba', Archivos de Zootecnia, vol. 60, no. 231, pp. 513-520.

Kadarmideen, HN, Thompson, R, Coffey, MP \& Kossaibati, MA 2003, 'Genetic parameters and evaluations from single- and multiple-trait analysis of dairy cow fertility and milk production', Livestock Production Science, vol. 81, pp. 183-195.

Kaiser, HF 1960, 'The application of electronic computers to factor analysis', Educational and Psychological Measurement, vol. 20, no. 1, pp. 141-151, viewed 18 Jan 2018, <https://doi.org/10.1 $177 / 001316446002000116>$

Khan, TA, Tomar, AKS, DUTT, T \& Bhushan, B 2013, ‘Principal component regression analysis in lifetime milk yield prediction of crossbred cattle strain Vrindavani of North India', The Indian Journal of Animal Sciences, vol. 83, no. 12, pp. 1288-1291.

Ledic, IL, Tonhati, H, Verneque, R da S, El Faro, L, Martínez, ML, Nápolis, C, Campos, JC, de Oliveira, L \& Galvão, L 2002, 'Genetic, phenotypic and environmental parameters for milk production at the control day and 305 days of lactation of Gir breed cows', Revista Brasilera de Zootecnia, vol. 31, no. 5, pp. 1953-1963. 
Oliveira, DP, Barros, CC, Neto, FRA, Lourenco, DAL, Hurtado-Lugo, NA \& Tonhati, H 2014, 'Principal Components for Reproductive and Productive Traits in Buffaloes from Brazil', 10th World Congress of Genetics Applied to Livestock Production. Vancouver pp.3-5, viewed 12 Feb 2015, <https://asas.org/docs/defaultsource/wcgalp-posters/611_paper_8988_manuscript_1420_0. pdf?sfursn=2>.

Ojango, JM \& Pollott, GE 2001, 'Genetics of milk yield and fertility traits in Holstein-Friesian cattle on large-scale Kenyan farms', Journal of Animal Science, vol. 79, no. 7, pp. 1742-50.

Ojango, JM, Ducrocq, V \& Pollott, GE 2005, 'Survival analysis of factors affecting culling early in the productive life of Holstein-Friesian cattle in Kenya', Livestock Production Science, vol. 92, no. 3, pp. 317-322.

Palacios-Espinoza, A, Espinoza Villavicencio, JL, González-Peña, D, Guerra Iglesias, D, De La Peña, RL \& Rodríguez Almeida, F 2007, 'Estimation of covariance components for the first four lactations in Holstein cattle according to different models', Zootecnia Tropical, vol. 25, pp. 9-18, viewed 12 Jan 2018, <http://www.ceniap. gov.ve/pbd/RevistasCientificas/ZootecniaTropical/zł2501/arti/ palacios\%20a.htm>.

Portales, A, González-Peña, D, Guerra, D \& Ortiz, J 2007, 'Parámetros genéticos para rasgos reproductivos de primer parto en el genotipo Siboney de Cuba', Revista Ciencia y Tecnología Ganadera, vol. 1, pp. 143- 146.

Rencher A C 2002, 'Methods of multivariate analysis'. Wiley-Interscience: New York.

Ribas, M, Gutiérrez, M, Mora, M, Evora, JC \& Gonzáles, S 2004, 'Comportamiento productivo y reproductivo del Siboney de Cuba en dos localidades', Cuban Journal of Agricultural Science, vol. 38, no. 2, pp. 121- 125 .

Ríos-Utrera, A, Hernández-Hernández, VD, Villagómez Amezcua-Manjarréz, E \& Zárate-Martínez, JP 2013, 'Heredabilidad de características reproductivas de vacas Indubrasil', Agronomía mesoamericana, vol. 24, no. 2, pp. 293-300.
Ruiz-Sánchez, R, Blake, RW, Castro-Gómez, HMA, Sánchez, F, Montaldo, HH \& Castillo-Juárez, H 2007, 'Short communication: Changes in the association between milk yield and age at first calving in Holstein cows with herd environment level for milk yield', Journal of Dairy Science, vol. 90, no.10, pp. 4830-4834.

Santana, I, Guerra, D, Veliz, D, Falcón, R, Rodríguez, M, González-Peña, D \& Ortiz J 2004, 'Parámetros genéticos y no genéticos que afectan las características reproductivas de la hembra Cebú en Cuba', Revista Cubana de Reproducción Animal, vol. 30, pp. 39-46.

SAS 2010, 'User's Guide Statistics', SAS Institute Inc,Cary, NC.USA.

Smith, HF 1936, 'A discriminant function for plant selection', Ann. Hum. Genet, vol. 7, pp. 240-250.

SPSS Inc 2002, 'User's Guide', SPSS Inc., Chicago.

Suárez, MA \& Pérez, T 2005, 'Incorporación a la reproducción en novillas Siboney de Cuba. Parámetros genéticos', CD-ROM, III Congreso Internacional de Mejoramiento Animal, 7-1 1 de noviembre, La Habana, Cuba, ISBN 959-7164-67-1 (MA-98).

Valle, A \& Moura FA 1986, 'Herencia de los principales parámetros productivos y reproductivos en vacas mestizas (5/8 Pardo Suizo $3 / 8$ criollo) tipo Carora', Zootecnia Tropical, Vol. 4, no. 1 y 2, pp. 49-65, viewed 25 jun 2004, <http://www.ceniap.gov.ve/bdigital/ ztzoo/zt0412/texto/herencia.htm>.

Van Vleck, LD 1987, 'Contemporary groups for genetic evaluations', Journal of Dairy Science, vol. 70, pp. 2456-2464.

Veliz, D, Guerra, D, Falcón, R, Santana, I, Rodríguez, M \& GonzálezPeña, D 2004, 'Parámetros genéticos y no genéticos que afectan la reproducción en la raza Santa Gertrudis', Revista Cubana de Reproducción Animal, vol. 30, pp. 53-58.

Vercesi Filho, AE, Madalena, FE, Albuquerque, LG, Freitas, AF, Borges, LE, Ferreira, JJ, Teodoro RL \& Faria FJC 2007, 'Parâmetros genéticos entre características de leite, de peso e a idade ao primeiro parto em gado mestiço leiteiro (Bos taurus $\times$ Bos indicus)', Arquivo Brasileiro de Medicina Veterinária e Zootecnia, vol. 59, pp. 983-990. 